\title{
Capturing of Real Image of Neck-Like Deformation from High-Speed Melt Spinning Line
}

\author{
Takeshi Kikutani ${ }^{* 1}$, Yutaka Kawahara*2, Naoki Ogawa, and Norimasa Okui \\ Department of Organic and Polymeric Materials, Tokyo Institute of Technology \\ 2-12-1, Ookayama, Meguro-ku. Tokyo, 152 Japan
}

\begin{abstract}
Observation of high-speed spinline using a video camera was carried out to obtain directly the detailed diameter profiles of neck-like deformation. An optical system was developed by adding a set of cylindrical lenses to the usual telescope-type optical system. This system magnified the horizontal direction four times larger than that in the vertical direction. Using this apparatus, the image of the neck-like deformation in high-speed splinline of poly (butylene terephthalate) was successfully captured. Although the shape of the neck-like deformation was more moderate than that of the "necking" observed in the colddrawing of undrawn fibers, the calculated velocity gradient at the end point of the neck-like deformation showed extremely high values of ca.10 and $30 \mathrm{~ms}^{-1}$ for the take-up velocities of 4 and $5 \mathrm{~km} / \mathrm{min}$, respec. tively.
\end{abstract}

\section{Introduction}

Fifteen years have elapsed since the bottle-neck type fiber diameter attenuation, i.e. "neck-like deformation", was found in the high-speed melt spinning line of poly (ethylene terephthalate) [ 1 ]. Using different on-line measuremental techniques, the presence of the neck-like deformation was confirmed in high-speed spinline of various crystalline polymers, such as poly (ethylene terephthalate) $[2,3]$, poly (butylene terephthalate) [4], polyamide $6[5,6]$. polyamide $66[6]$, polyamide 12 $[7]$, and poly (phenylene sulfide) [ 8$]$. Intensive efforts have been made to elucidate the mechanism of this diameter change in the spinline $(9,10)$, since the major part of fiber structure develops with the neck-like deformation. The control of the neck-like deformation is believed to be essential for the improvement of the structure and properties of high-speed spun fibers.

We have carried out diameter and temperature measurements of the spinline paying particular attention to the vicinity of the neck-like deformation. Through the

\footnotetext{
*1 To whom correspondences should be addressed

* 2 Present address : The Tokyo Metropolitan Textile Re. search Institute
}

spinline temperature measurements [11], the heat evolution accompanied with the orientation-induced crystallization near the neck-like deformation was detected. At the same time, the crystallization rate in the high-speed spinline was estimated. The diameter profiles of the neck-like deformation was analyzed in detail by combining the signals from two diameter monitors [12]. Our conclusion was that the sharpness of the neck-like deformation increases with the take-up velocity, however, the maximum velocity gradient was much smaller than the value which we expected from the shape of so-called "necking" observed in the cold-drawing of undrawn fibers. Haberkorn et al. (6) also have measured the detailed diameter profile of polyamide 66 spinline in the vicinity of the neck-like deformation using the laser light scattering technique, and reported a relatively mild diameter attenuation. Although these results were from on-line diameter measurements, the information was in direct because data processing was carried out under some assumptions.

In this study, we have tried to obtain the image of the neck-like deformation directly using a video camera. To the knowledge of the authors, there has been no report. in which the real image of the neck-like deformation is captured in the high-speed spinning process. 


\section{Experimental}

\subsection{High-Speed Melt Spinning}

High-speed melt spinning of poly (butylene terephathalate) (PBT) (Teijin Limited) was conducted using a melt spinning system comprised of an extruder with a screw diameter of $25 \mathrm{~mm}$, a gear pump and a take-up de. vice. Molten polymer was extruded through a spinneret of $1 \mathrm{~mm}$ diameter at a mass out flow rate of $5.5 \mathrm{~g} / \mathrm{min}$. The extrusion temperature was $255^{\circ} \mathrm{C}$. The take-up device was located at $330 \mathrm{~cm}$ below the spinneret and the extruded filament was taken-up at velocities of 4 and 5 $\mathrm{km} / \mathrm{min}$. The melt spinning system was also equipped with a stage which was movable along the spinline. The recording of the image of running filament was done by setting a measuring apparatus on the stage.

\subsection{Measuring Apparatus}

The image of the neck-like deformation was difficult to be captured in the spinline by two reasons, i. $e$. the posi tion of neck-like deformation fluctuates up and down along the spinline, and the fiber position also fluctuates in the direction perpendicular to the filament axis.

In the previous paper (12), we have reported that the velocity gradient near the end point of the neck-like deformation of PBT went up to ca. $20 \mathrm{~ms}^{-1}$ when the take-up velocity was $5 \mathrm{~km} / \mathrm{min}$. This means that if the image of a filament is captured with the camera's field of view of $2 \times 2 \mathrm{~mm}$, the maximum diameter change in the field is expected to be about $40 \%$ of the final diameter. This might be detectable, even considering the severity of the measurement condition, if a proper magnification is chosen. We also have reported, however, that the position of neck-like deformation fluctuates along the spin. line at an average speed of ca. $1 \mathrm{~m} / \mathrm{s}$. Since a standard video cassette recorder records 30 frames per second. this result implies that the neck-like deformation moves about $30 \mathrm{~mm}$ between two consecutive frames. Under these conditions, it can be understood that the neck-like deformation is not easy to be captured using a video camera.

To overcome the above difficulties, we developed an optical system in which a set of convex and concave cylindrical lenses were added in an afocal manner to the standard telescope-type optical system. With these extra lenses, the image was additionally magnified only in the horizontal direction. Schematic illustrations of the ex

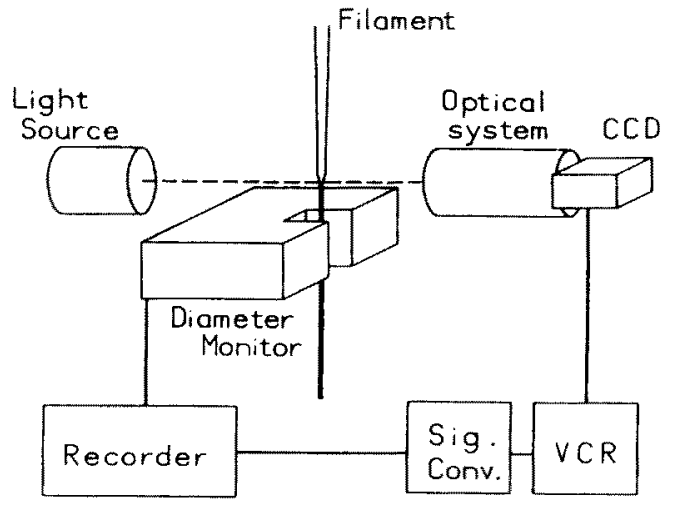

(a)

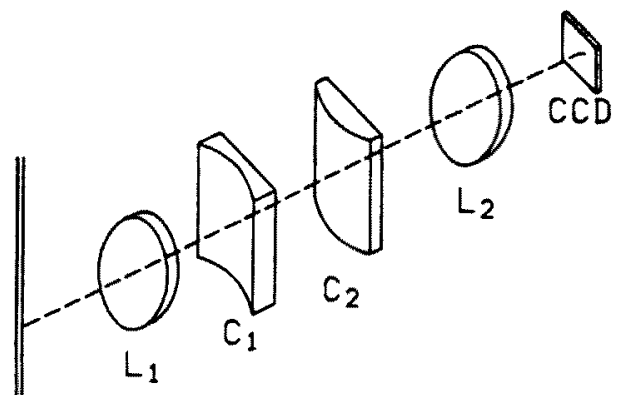

Filament

(b)

Fig. 1 (a) Schematic diagram of filament image record. ing system. (b) Optical system in the apparatus: $L_{1}$ and $L_{2}$, circular convex lenses; $C_{1}$ and $C_{2}$, cylindrical convex and concave lenses.

perimental set-up and the optical system used in this study are shown in Figs. 1-(a) and (b).

In this experiment, a diameter monitor (Zimmer OHG, Model $460 \mathrm{~A} / 2$ ), which has measurement range of $0-2$ $\mathrm{mm}$, was set at the focal length of the video-camera optical system. Along with the filament image, the information whether the signal from the diameter monitor exceeded a user-defined cut-off value or not was recorded on the audio track of the video cassette and was utilized for judgement of focusing.

The detection of the filament image depends on the contrast between the filament and the back ground. In our system, the filament was illuminated from the opposite side of the video camera. The recorded image was analyzed using a personal computer equipped with an image processing card (Photron, Image digitizer FRM2512) 


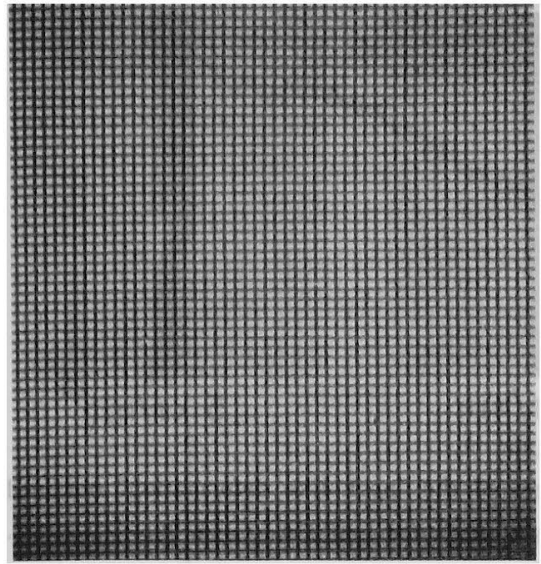

( a )

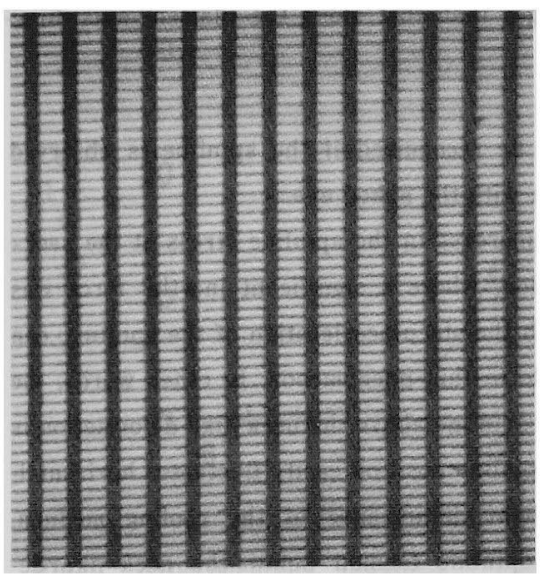

Fig. 2 Images of wire mesh recorded (a) without cylindrical lenses, and (b) with cylindrical lenses.

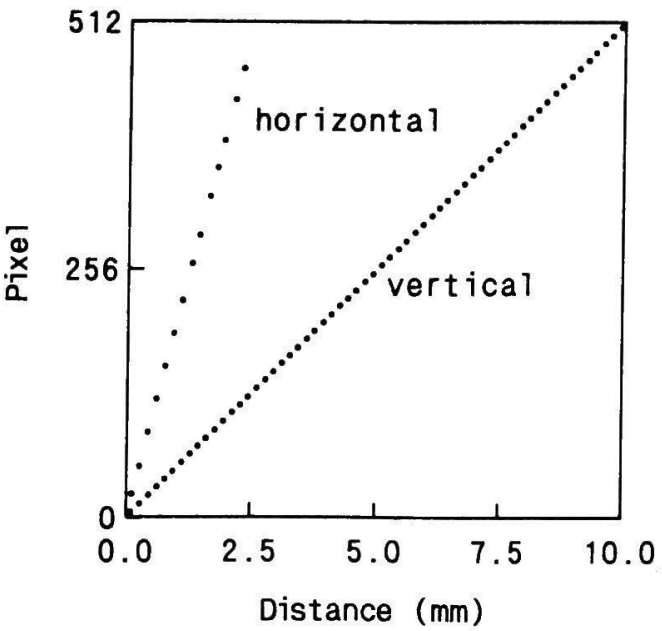

Fig. 3 Relation between distances in the real space and pixels in the field of view analyzed from the image shown in Fig.2-(b).
For the measurement of the overall high-speed spinline profiles, the diameter monitor described above was set on the movable stage, and filament diameter was measured every ten centimeters along the spinline from 40 to $220 \mathrm{~cm}$ below the spinneret.

\section{Results and Discussion}

\subsection{Optical System}

To demonstrate the function of cylindrical lenses, images of a wire mesh with wire diameter of $63 \mu \mathrm{m}$ and wire to wire distance of $165 \mu \mathrm{m}$ were obtained using the optical system described above. The images recorded with and without the cylindrical lenses are compared in Figs.2-(a) and (b). It can be recognized from the image obtained with cylindrical lenses (Fig.2-(b)) that the magnification in the horizontal direction is larger than that in the vertical direction.

In Fig.3, the positions of the center of wire in the image field shown in Fig.2-(b) were plotted against the distance in the real space. It was confirmed from this figure that the area of ca. $2 \times 10 \mathrm{~mm}$ can be captured by this optical system, and from the slope of the plots for the vertical and horizontal directions, the ratio of magnification between two directions was calculated to be as 4.0. The linear relation of the plotted data also confirmed that there was not any significant distortion of the image in the field of view.

\subsection{Neck-Like Deformation}

Filament diameter profiles of the high-speed spinline measured at the take-up velocities of 4 and $5 \mathrm{~km} / \mathrm{min}$ are shown in Fig.4. Abrupt diameter attenuation, i.e. the neck-like deformation, was observed at $150-160 \mathrm{~cm}$ for $4 \mathrm{~km} / \mathrm{min}$ and at $130-140 \mathrm{~cm}$ for $5 \mathrm{~km} / \mathrm{min}$.

Five consecutive video frames extracted from the recorded spinline images for the take-up velocity of 5 $\mathrm{km} / \mathrm{min}$ and at the position of $140 \mathrm{~cm}$ are shown in Figs.5-(a)-(e). From Figs.5-(a) and (e), it can be seen that, though there is no significant diameter variation within each frame, there is an obvious increase in filament diameter from (a) to (e). This result implies that the position of the neck-like deformation moved downward through the camera's field of view, and it can be seen in Fig.5-(c) that there is a significant diameter difference between the top and bottom part of the filament image.

In order to show more distinctly the diameter profiles 


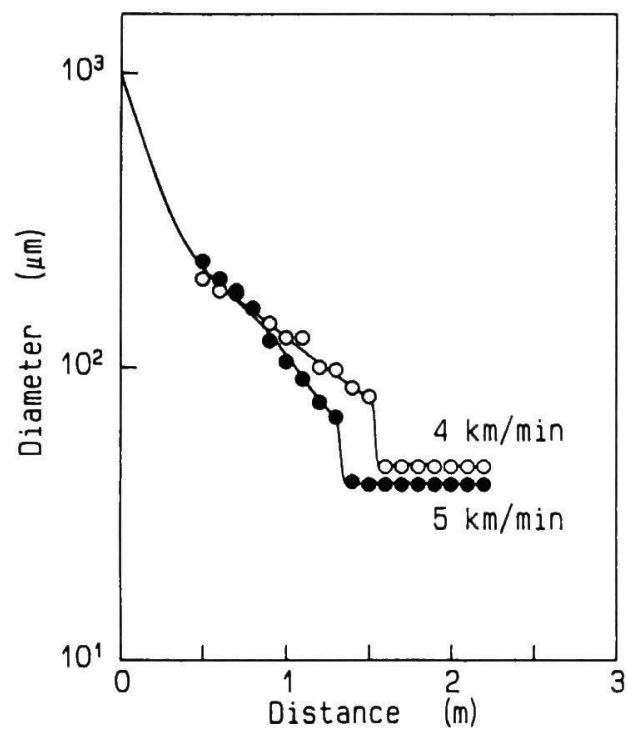

Fig. 4 Diameter profiles of high-speed spinline for poly (butylene terephthalate) measured at take-up velocites of 4 and $5 \mathrm{~km} / \mathrm{min}$.

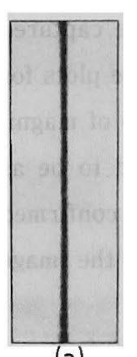

(a)

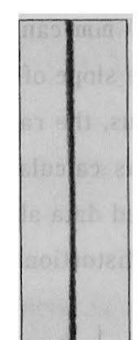

(b)

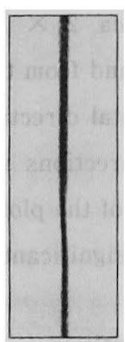

(c)

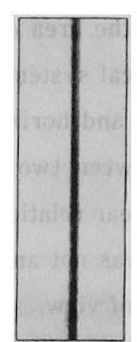

(d)

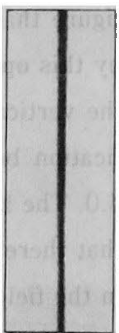

(e)
Fig. 5 Five consecutive frames of filament image obtained through on-line measurement at a take-up velocity of $5 \mathrm{~km} / \mathrm{min}$.

in the vicinity of neck-like deformation, the ratio of the magnification of the captured images obtained in this manner was changed to $1: 16$ (=vertical : horizontal) using the image processing technique. The results for the take-up velocities of 4 and $5 \mathrm{~km} / \mathrm{min}$ are shown in Figs. $6-(a)$ and (b). In each figure, there obviously is a sharp diameter attenuation in the middle part of the filament image.

A slight bending of the filament at the end point of the neck-like deformation was observed in both results. It must be noted that the bending behavior is emphasized in these figures because of the higher magnification in the horizontal direction. In practice, however, such a phe-

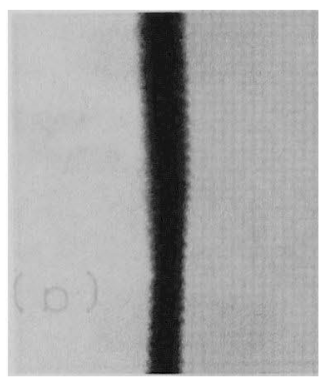

$4 \mathrm{~km} / \mathrm{min}$

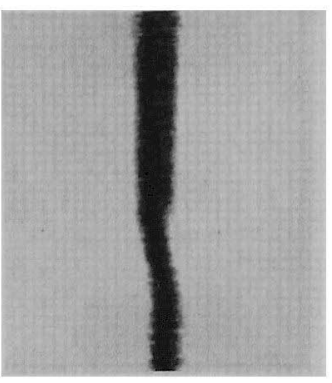

$5 \mathrm{~km} / \mathrm{min}$
Fig. 6 Images of the "neck-like deformation" obtained at take-up velocities of 4 and $5 \mathrm{~km} / \mathrm{min}$. The magnification in the direction perpendicular to the filament axis is 16 times larger than that along the filament axis direction.

nomenon can be recognized if the spinline is observed carefully even with the naked eye.

An abrupt increase in the spinline stress at the necklike deformation, where a significant inertial force was generated with the steep acceleration of the spinline, can be attributable to this phenomenon. In other words, the tension of the spinline above the neck-like deformation is low and the spinline position easily fluctuates horizontally with a slight disturbance of the cooling medium, whereas the spinline tension below the neck-like deformation became higher and the spinline tends to be straighten-up and run vertically. The fact that the diameter profiles measured above the neck-like deformation position in the high-speed spinline, especially when the mass out flow rate is relatively high, do not show significant difference with the take-up velocity and behave like a freely falling polymer liquid might be the evidence for the low spinline tension in this region (2-8].

\subsection{Velocity Gradient}

Optical density distribution curves in the direction perpendicular to the filament axis were prepared from the images shown in Fig.6. By assuming a proper threshold value, the variation of filament diameter along the filament axis was analyzed as shown in Figs. 7 and 8. Diameter profiles obtained by fitting a mathematical formula proposed in the previous paper [12] to the data, and the velocity gradient profiles calculated using the equation of continuity are also plotted in the figures. The diameters after the neck-like deformation were in fair agreement with those of as-spun fibers. 


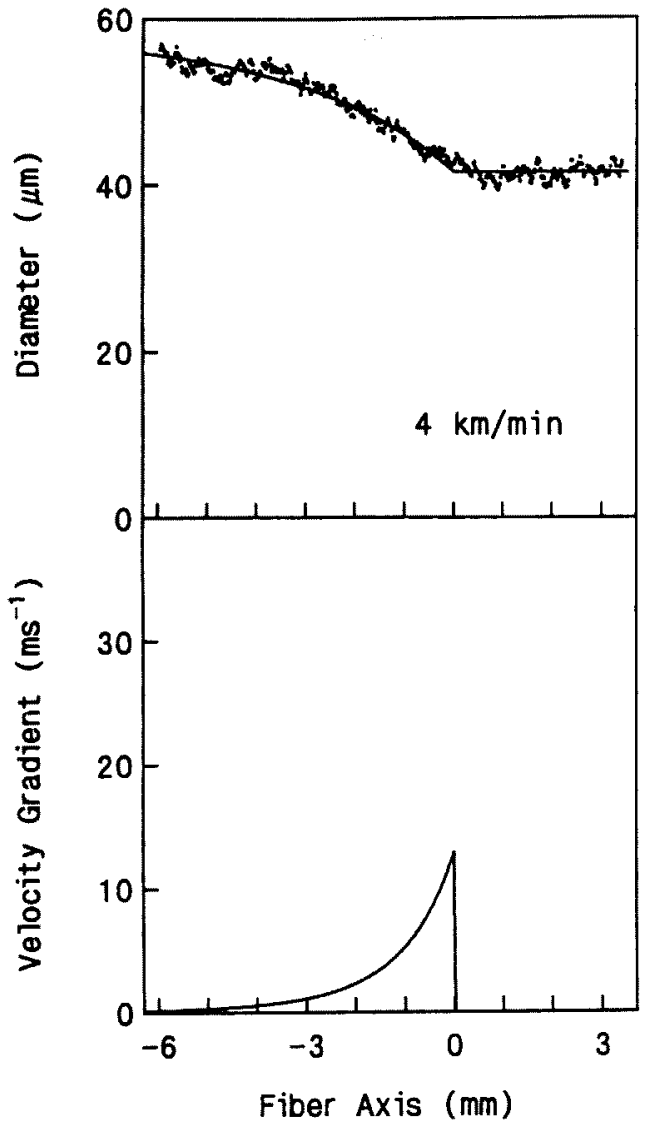

Fig. 7 Experimentally obtained (closed circle) and analyzed (solid line) diameter profiles, and velocity gradient profiles in the vicinity of neck-like deformation for take-up velocity of $4 \mathrm{~km} / \mathrm{min}$. Zero on the abscissa corresponds to the end point of neck-like deformation.

The diameter attenuation accelerated monotonically as the deformation proceeded to the end point of the necklike deformation. Accordingly, the velocity gradient increased continuously up to the end point of the neck-like deformation, and reached ca. 10 and $30 \mathrm{~ms}^{-1}$ for the take-up velocities of 4 and $5 \mathrm{~km} / \mathrm{min}$, respectively. These velocity gradient values agreed well with those obtained in the previous paper (12). At a take-up velocity of $1 \mathrm{~km} / \mathrm{min}$, for example, the maximum velocity gra. dient. which naturally depends on other spinning conditions and polymer characteristics, is known to be several tens of reciprocal seconds [2]. The velocity gradient obtained in this study was about three orders of magnitude larger, and was proved to be an extremely large value.

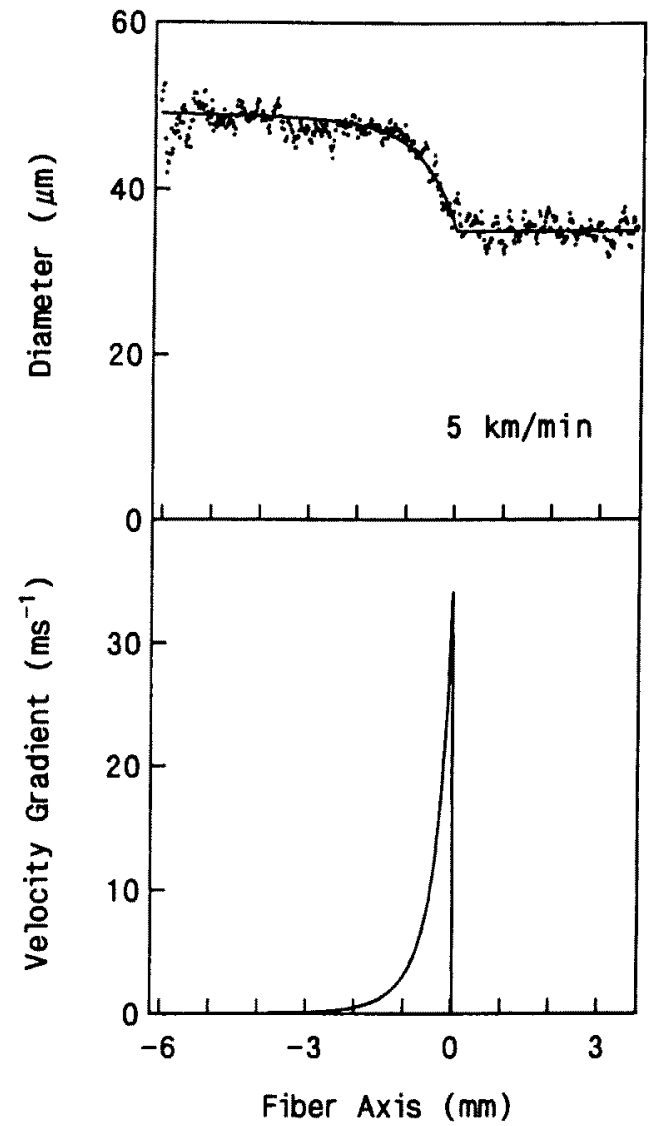

Fig. 8 Experimentally obtained (closed circle) and analyzed (solid line) diameter profiles, and velocity gradient profiles in the vicinity of neck-like deformation for take-up velocity of $5 \mathrm{~km} / \mathrm{min}$. Zero on the abscissa corresponds to the end point of neck-like deformation.

For the sake of more intuitive understanding of the neck-like deformation characteristics, the diameter profiles were replotted adopting the same magnification in the directions parallel and perpendicular to the filament axis in Fig.9. The arrows in the figure indicate the end point of the neck-like deformation. This figure confirms that the actual shape of the neck-like deformation in the high-speed spinline is so moderate that it appears rather difficult to find the end point of deformation clearly. From this result, the authors are considering that the mechanism for the occurrence of the neck-like deformation should be explored under the assumption of the simple uniaxial elongation. Further investigation on the change of detailed diameter profile with the spinning con ditions and polymer characteristics might be necessary 


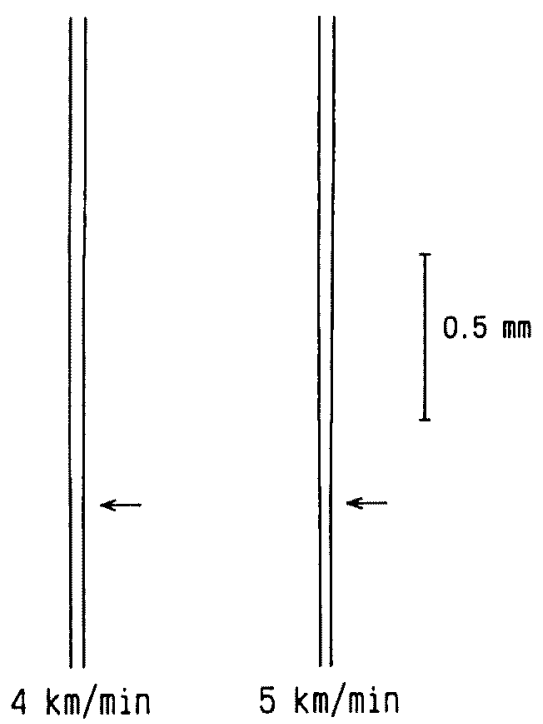

Fig. 9 Neck-like deformation profiles depicted with the same magnification in the directions parallel and perpendicular to the filament axis.

for the deeper understanding of the mechanism of necklike deformation.

\section{Acknowledgement}

This work was supported by a Grant in Aid for Scien. tific Research (No.05650915) from the Ministry of Education, Japan.

\section{References}

1. G. Perez and C. Lecluse, Proc. 18th Int. Man made Fibre Conf., Dornbirn, Austria 1979.

2. J. Shimizu, N. Okui, and T. Kikutani, "High-speed Fiber Spinning" (A. Ziabicki and H. Kawai Eds.), John Wiley \& Sons, p. 173 (1985).

3. K. Fujimoto, K. Iohara, S. Owaki, and Y. Murase, Sen'i Gakkaishi, 44, 53 (1988).

4. S. Chen, W. Yu, and J. E. Spruiell, J. Appl. Polym. Sci., 34, 1477 (1987).

5. J. H. Behda and J. E. Spruiell, J. Appl. Polym. Sci, 39 447 (1990).

6. H. Haberkorn, K. Hahn, H. Breuer, H.-D. Dorrer, and P. Matthies, J. Appl. Polym, Sci., 47, 1551 (1993).

7. J. Shimizu, Sen'i-kikai Gakkaishi, 44, 317 (1985).

8. T. Kikutani, K. Wakayama, A. Takaku, and J. Shimizu, Sen'i Gakkaishi, 48, 549 (1992).

9. A. Ziabicki, J. Non-Newtonian Fluid Mech., 30, 141 (1988).

10. A. Ziabicki and J. Tian, J. Non-Newtonian Fluid Mech., 47, 57 (1993).

11. T. Kikutani, Y. Kawahara, T. Matsui, A. Takaku, and J. Shimizu, Seikei-Kakou, 1, 333 (1989).

12. T. Kikutani, T. Matsui, A. Takaku, and J. Shimizu, Sen'i Gakkaishi, 45, 441 (1989). 